\title{
Rare Kaon Decays at LAMPF II
}

T. W. L. Sanford*

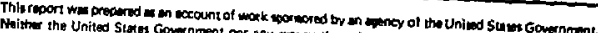

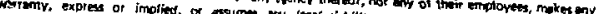

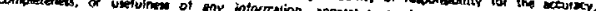

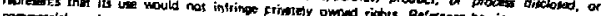

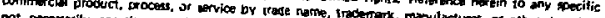

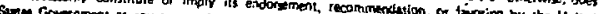

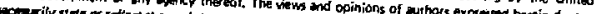

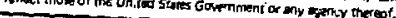

*Sandia National Laboratorles, Albuquerque, NM 87185.

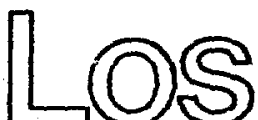


RARE KAON DECAYS AT LAMPF II

by

T. W. L. Sanford

\begin{abstract}
At LAMPF II, intense beams of kaons will be avallable that will enable the rare kaon-decay processes to be investigated. This note explores some of the possibilities, which divide into two classes: (1) those that test the standard model of Inberg and Salam and (2) those that are sensitive to new interactions. For both classes, experiments have been 1 imited not by systematic errors but rather by statistical ones. LAMPF II with its intense flux of kaons thus will enable the frontler of rare kaon decay to be reallstically probed.
\end{abstract}

\title{
I. INTRODUCTION
}

The study of kaon decay has made a major contribution to our understanding of the interaction between elementary particles. The resolution of the $\theta-\tau$ puzzle in 1956, for example, led to the discovery of parity violation in the weak interaction with the suggestion that CP was the correct symatry. The observation of the sinall CP-violating decay $K_{L} \rightarrow \pi^{+}{ }^{-}$in 1964, however, removed the concept that CP is an exact symmetry of nature. During the early 1970s, the observation of no significant strangeness-changing neutral current as exemplified by the strong suppression of $\mathrm{K}_{\mathrm{L}} \rightarrow \mu^{+} \mu^{-}$decays led in the framework of unified gauge theories to the introduction of the charm quark. This quark was subsequently discovered in 1974 .

At present, experimental data are consistent with the $s U(2)_{L} \times U(1)$ gauge model of WeInberg and Salam (WS) that unifies the electromagnetic and weak interaction. 1 studying the rare decay modes of the kaon enables important 
detalls of the model to be tested. The model may, however, be limited in 1 ts. description of the electroweak interaction. The model offers, for example, no explanation or differentiation among the three generations of leptons and quarks observed in nature. New interactions, which would link these generations, are not contained in the model. Some rare decays of the kaon are sensitive to such interactions. Most importantly, the measurenent of these decays offers perhaps the only way of probing interactions in multi-TeV energy regions.

The physics to be investigated requires precision measurements of sall corrections to parameters of known processes. In addition, measurements of small but finite branching ratios for decays, which under some selection rule might not be allowed, are needed. As such, progress has been 11mited by the lack of adequate kaon fluxes.

The 100-fold increase in primary proton fius expected at LAMP II over existing facilities wil provide ample kaon fluxes that wil enable experimenters both to confront the WS model significantly and to probe for new interactions not contained in the model. With such a facility, developients comparable with those of the past two decades can be expected.

The types of decays that can be studied in such a facility divide into two categories: (1) those that test the standard model of WS and (2) those that are sensitive to new interactions not contained in the model. Before the two classes are discussed together with experimental examples, the flux of kaons and the sensitivity to these decays at LAMPF II are investigated.

\section{KAON FLUX}

Experiments to measure kaon decays have been performed using both decays from kaons decaying in flight (in-flight beam) and decays from kaons stopping in a target (stopping beam).

The production cross section for $\mathrm{K}^{+}$and $\mathrm{K}^{-}$mesons generated at zero degrees by $16-\mathrm{GeV} / \mathrm{c}$ incident protons on a nuclear target ${ }^{2}$ increases 1 ike $P_{k}{ }^{2}$ at low kaon momentum and peaks at $5 \mathrm{GeV} / \mathrm{c}$ and $3 \mathrm{GeV} / \mathrm{c}$ for $\mathrm{K}^{+}$and $\mathrm{K}^{-}$, respectively (Fig. I). Optimum useful yields of kaon decays occur at about $600 \mathrm{MeV} / \mathrm{c}$ for stopping beans and at about $6 \mathrm{GeV} / \mathrm{c}$ for in-flight beams.

Assuming beam-line characteristics similar to the LESB II and MESB beans currently operating at the Brookhaven National Laboratory Alternating-Gradient Synchrotron ${ }^{3}$ 


\begin{tabular}{c|c}
\hline STOPPING BEAM & IN-FLIGHT BEAM \\
\hline LESB II & MESB \\
$\frac{\Delta P}{\mathbf{P}}= \pm 2.5 \%$ & $\frac{\Delta P}{\mathbf{P}}= \pm 37$ \\
$\Delta \Omega=15 \mathrm{MSR}$ & $\Delta \Omega=0.3 \mathrm{MSR}$ \\
$\mathrm{L}=15 \mathrm{M}$ & $\mathrm{L}=45 \mathrm{M}^{\star}$
\end{tabular}

and detection efficiencles of

$E F F=50 \% \quad E F F=15 \%$

the following measurable yields of kaor decays in a 100-day run with $10^{14}$ Interacting protons per second can be expected at LAMPF II: ${ }^{4}$

\begin{tabular}{|ccc|}
\hline & STOPPING BEAM & IF-FLIGHT BEAM \\
\hline $\mathrm{K}^{-}$ & $7 \times 10^{13}$ & $1 \times 10^{14}$ \\
$\mathrm{~K}^{+}$ & $3 \times 10^{14}$ & $7 \times 10^{14}$ \\
\hline
\end{tabular}

At least a factor of two increase in these rates is anticipated from inproved beam-1ine design employing superconducting magnets with combined functions.

Using the estimated number of decays from the in-filght $\mathbf{K}^{+}$beam, a statistical linit of $1.4 \times 10^{-15}$ on a branching ratio for a particular channel can be placed. The large number of measurable kaon decays thus beconea powerful tool for sensitively examining rare-decay processes.

* The noninal length of 81 a 1 s assumed shortened to 45 ql. 


\section{DECAYS THAT TEST STANDARD WS MODEL}

Two classes of decays that put fundamental constrafnts on the standard model of WS are (1) those that test $\mu$-e universality and (2) those that test the Glashow-I1lopoulous-Mainani (GIM) cancellation wechanism in strangeness-changing neutral-current transitions.

\section{A. H-e Universality}

The V-A structure of the weak current and the assumption of $\mu-e$ universality are cornerstones of the older weak-interaction theory and of the newer renormaifition gauge theory of WS that unifies the electronagnetic and weak interaction. In the current-current description of the weak force, which is common to both theories, the decay of the kaon is expressed by a Lagrangian density of the form ${ }^{5}$

$$
L=\frac{G}{\sqrt{2}} \sin \theta_{c}\left\{\left\lfloor\psi_{\ell} \gamma_{\lambda}\left(1+\lambda_{5}\right) \psi_{v_{\ell}}\right] J_{\lambda}{ }^{\Delta S}+h c\right\},
$$

where the term in the square bracket is the $V$ - A form of the weak current, $J_{\lambda}{ }^{\Delta S}=1$ is the strangeness-changing hadronic current, $\left(l, \nu_{l}\right)$ is a lepton pair, $G$ is the Fermi coupling constant, and $\theta_{c}$ is the Cabbibo angle.

The V - A form of the weak current selecte opposite helicities for the $(l, v)$ pair in the limit of

$$
\frac{v}{c}+1
$$

for the charged lepton $\ell$. The decay

$$
\mathrm{x}^{+}+\mathrm{e}^{+} v_{\mathrm{e}}
$$

is therefore strongly suppressed relative to

$$
\mathrm{K}^{+}+\mu^{+} \nu_{\mu}
$$

If the weak current also contained a $V+A$ term, then lepton pairs of the same 
helicity would be allowed and the rate of $\mathrm{k}^{+} \rightarrow \mathrm{e}^{+} v_{e}$ relative to $\mathrm{k}^{+} \rightarrow \mu^{+} v_{\mu}$ would increase dramatica11y.

On the assumption of equal couplings for the $e$ and $\mu$ lepton pairs and the above form for the Lagrangian, the rate for $\mathrm{k}^{+}+\mathrm{e}^{+} v_{\mathrm{e}}$ relative to $\mathrm{k}^{+}+\mu^{+} v_{\mu}$ depends to first order only on the following ratio of masses:

$$
R_{K}=\frac{\Gamma\left(K^{+}+e^{+} v_{e}\right)}{\Gamma\left(K^{+}+\mu^{+} v_{\mu}\right)}=\left(\frac{M_{e}}{M_{\mu}}\right)^{2}\left(\frac{M_{K}^{2}-M^{2}}{M_{K}{ }^{2}-M_{\mu}{ }^{2}}\right)^{2}=2.57 \times 10^{-5} .
$$

The measurement of this ratio thus provides a fundamental test of both the $\mathrm{V}-\mathrm{A}$ interaction and $\mu-e$ universality for $\Delta S=1$ hadronic currents.

The test is analogous to the one that checks the theoretical ratio

$$
R_{\pi}=\frac{\Gamma\left(\pi^{+} \rightarrow e^{+} \nu_{e}\right)}{\Gamma\left(\pi^{+}+\mu^{+} \nu_{\mu}\right)}=\left(\frac{M_{e}}{M_{\mu}}\right)^{2}\left(\frac{M_{\pi}{ }^{2}-M^{2}}{M_{\pi}{ }^{2}-M_{\mu}{ }^{2}}\right)^{2}=1.28 \times 10^{-4}
$$

for $\Delta S=0$ hadronic currents.

Any deviation from either ratio would imply evidence for new effects, which will be discussed in Sec. IV.

$\mathrm{K}^{+}+\mathrm{e}^{+} v_{\mathrm{e}}$

$\mathbf{R}_{K}$ has recently been measured by Heintze and collaborators at the CERN-PS by stopping a beam of $\mathrm{K}^{+}$in a scintillator target. ${ }^{6}$ They used a gas Cerenkov counter to identify the positron and a set of drift chambers on either side of a bending magnet to measure its momentum. Part of the apparatus was surrounded by lead-glass counters in order to detect $\gamma$ rays from the background decay

$$
\mathrm{k}^{+}+\pi^{0} \mathrm{e}^{+} \nu_{\mathrm{e}}
$$


and

$$
\mathrm{k}^{+}+\mathrm{e}^{+} \mathrm{v}_{\mathrm{e}}^{\gamma}
$$

Two other sources of background were accidentals and

$$
k^{+}+\mu^{+} v_{\mu}
$$

decays. In this decay, the muon has a momentum of $236 \mathrm{MeV} / \mathrm{c}$.

The positron from the wanted tecay

$$
k^{+}+e^{+} v_{e}
$$

has a momentum of $247 \mathrm{MeV} / \mathrm{c}$. They observed 411 events whose momentum was greater than $240 \mathrm{MeV} / \mathrm{c}$. In this sample, only seven events were due to the above backgrounds. A correction of $32 \pm 1 \%$ was applied ta account for the loss of positrons having momentum below $240 \mathrm{MeV} / \mathrm{c}$ due to measuring resolution and bremstrahlung. Subtracting the small background and applying acceptance corrections they obtained

$$
R_{\text {exp }}=(0.98 \pm 0.06) R_{K}
$$

Here, the major fraction of the $6 \%$ error is simply the 5.27 statistical uncertainty on 411 events.

$\mathrm{K}^{+} \rightarrow \mathrm{e}^{+} \mathrm{ve}_{\mathrm{e}}$

A by-product of the experiment is the detection of the radiative structure-dependent (SD) decay of the kaon. 7,8

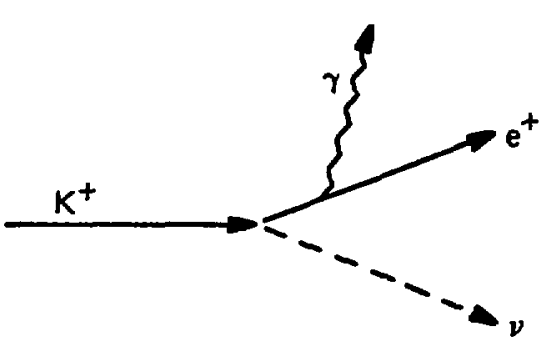

Internal Bremsstrahlung

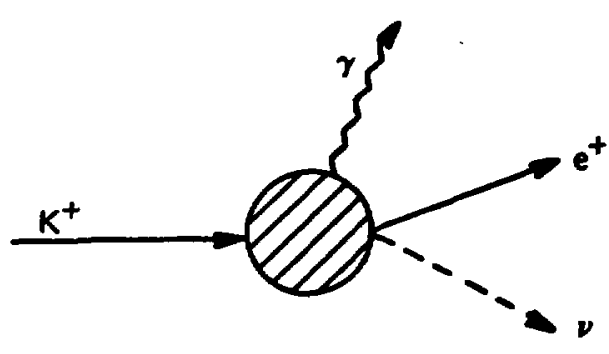

Structure-Dependent Decay 
The gelection rules allow the transition to be mediated by both the vector and axial-vector hadronic currents.

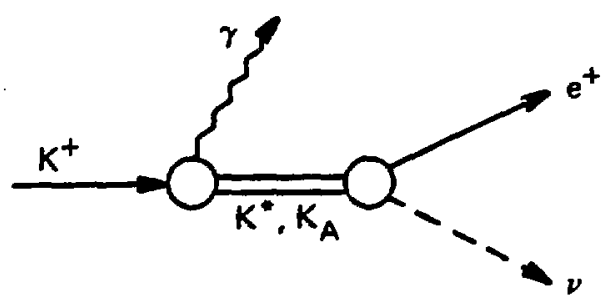

Accordingly, measurement of the transition gives information about the kaon vector $v_{K}$ and axial vector $a_{K}$ form factors. Besides $\mathrm{Ke}_{4}$ decays, this decay is the only other kaon decay whose measurement appears feasible that yields information simultaneously on both form factors.

The principal background for this process comes from $\mathrm{k}^{+} \rightarrow \pi^{0} \mathrm{e}^{+} v_{\mathrm{e}}$ decay, which has a branching ratio 3000 times that of $\mathrm{K}^{+}+\mathrm{e}^{+} \nu_{\mathrm{e}} \gamma$ decay. Measuring only events whose positron momentum is greater than $235 \mathrm{MeV} / \mathrm{c}$ suppresses the background and makes the radiative SD decay easy to identify. For this subclass of events,

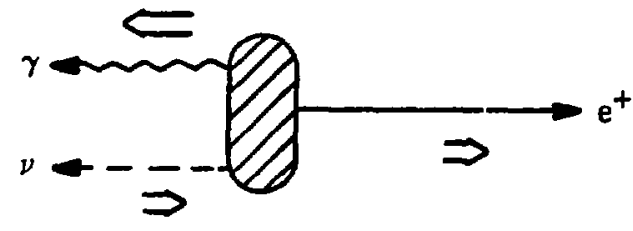

the helicity of the photon is positive, and the measured branching ratio for the process

$$
\frac{\Gamma\left(\mathrm{SD}_{+}\right)}{\Gamma\left(\mathrm{K}^{+}+\mu^{+} v_{\mu}\right)}=(2.33 \pm 0.42) \times 10^{-5}
$$

is sensitive to a linear combination of $v_{K}$ and $a_{K}$.

The data suggest that the decay is dominated by the $K *(892)$ vector transition, but the statistics are weak and much can be learned by refining the experiment. 


\section{B. GIM Cancellation Mechanisin}

The search for strangeness-changing neutra1-current decays has been essentially negative. Typically, 1 imits of $10^{-6}$ or less to a particular final state have been placed. These limits have had a profound impact on the understanding of the weak interaction because any renormalizable theory that unifies the electromagnetic and weak interaction contains not only charged currents but also neutral ones.

By introducing the charm quark, GIM have shown to first order that $\Delta S=1$ neutral-current transitions are not allowed but that $\Delta S=0$ neutril-current transitions are.

The allowed $\Delta S=0$ neutral currents have been found in neutrino interactions. They are difficult to observe in decay processes because any such transition can also proceed by the stronger electromagnetic force.

At present, two decays of the kaon with a $\Delta S=1$ and $\Delta Q=0$ transition have been observed. 8 They are

$$
\mathrm{K}^{+}+\pi^{+} \mathrm{e}^{+} \mathrm{e}^{-}
$$

and

$$
\mathrm{K}_{\mathrm{L}}+\mu^{+} \mu^{-}
$$

In both cases, the small branching ratio measured is consistent with a first-order weak process coupled with a first-order electromagnetic process rather than with a pure first-order neutral-current process.
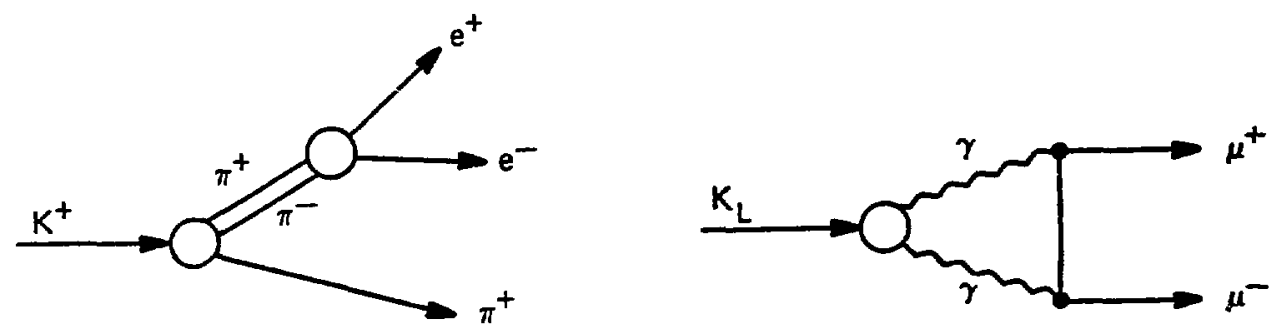

Decays such as the above are of interest for two reasons. First, they provide an excellent test of the GIM cancellation mechanisa. Second, they are sensitive to second-order electroweak effects, which in principle any renormalizable theory of the electroweak interaction can evaluate. 
The following is a list of such processes together with (1) the current experimental rates or bounds ${ }^{9}, 10$ and (2) the rate as calculated in 1974 by Gat1lard and Lee ${ }^{11}$ using the wS model.

PROCESS

EXPERIMENTAL

RATE OR BOUND
ORDER OF MAGNITUDE

OF BRANCHING RATIO
$\mathrm{x}_{\mathrm{L}}+\mu^{+} \mu^{-}$
$(9.1 \pm 1.9) \times 10^{-9}$
$10^{-8}$
$\mathrm{K}_{\mathrm{s}}+\mu^{+} \mu^{-}$
$<3.2 \times 10^{-7}$
$10^{-10}$
$\mathrm{K}^{+} \rightarrow \pi^{+} \nu \bar{\nu}$
$<5.6 \times 10^{-7}$
$10^{-10}$
$K_{\mathrm{L}}+\pi^{\circ} \nu \bar{v}$
$-$
Forbidden
$K_{s} \rightarrow \pi^{0} v \bar{v}$
$-$
$K_{L}+\gamma \gamma$
$(4.9 \pm 0.5) \times 10^{-4}$
$10^{-12}$
$\mathrm{x}_{\mathrm{s}} \rightarrow \mathrm{r}$
$<4 \times 10^{-4}$
$10^{-4}$
$\mathrm{K}^{+} \rightarrow \pi^{+} \gamma \gamma$
$<3.5 \times 10^{-8}$
$1.4 \times 10^{-6}$
$K_{L}+\pi^{o} \gamma \gamma$
$<2.4 \times 10^{-4}$
$10^{-6}-10^{-7}$
$\mathrm{K}_{\mathrm{s}}+\pi^{\mathrm{o}} \gamma \boldsymbol{\gamma}$
-
$\mathrm{K}^{+}+\pi^{+} \mathrm{e}^{+} \mathrm{e}^{-}$
$(2.6 \pm 0.5) \times 10^{-7}$
$<10^{-7}$
$\mathrm{K}_{\mathrm{L}} \rightarrow \pi^{0} \mathrm{e}^{+} \mathrm{e}^{-}$
$<2.3 \times 10^{-6}$
$10^{-8}-10^{-7}$
$K_{S} \rightarrow \pi^{0} e^{+} e^{-}$
$-$

$\underline{K}^{+}+\pi^{+} v \nabla$

The decay $\mathrm{x}^{+}+\pi^{+} \nu \bar{\nu}$ is particularly interesting to measure because no competing first-order weak and electromagnetic effects exist as they do in $\mathrm{K}^{+} \rightarrow$ $\pi^{+} e^{+} e^{-}$or $K_{L}+\mu^{+} \mu^{-}$decays.
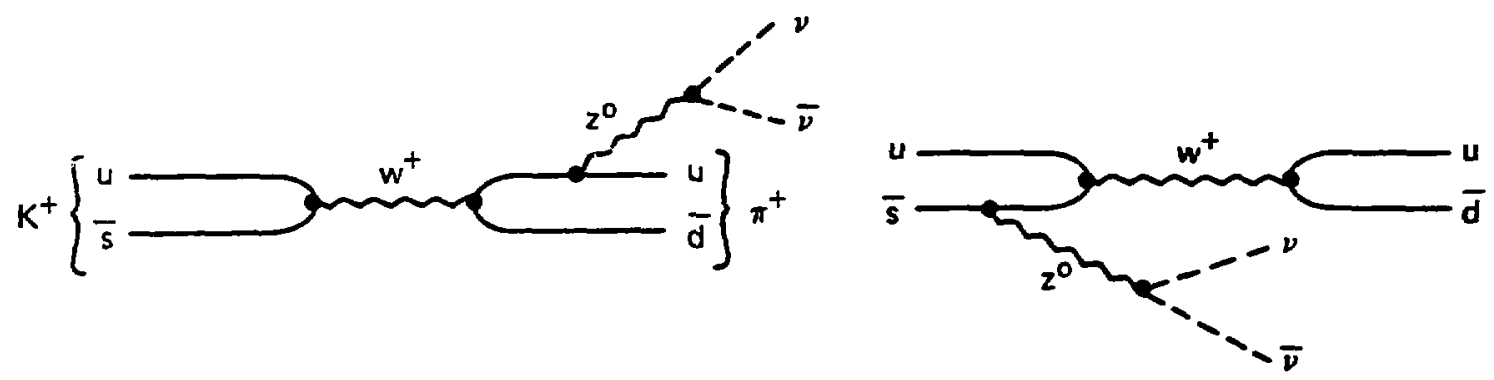

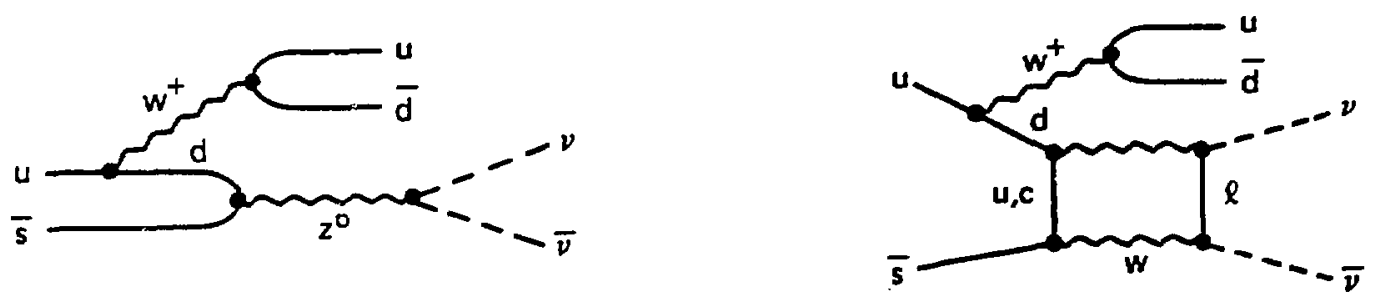

In 1978, Me and Okada ${ }^{12}$ showed that the branching ratio for this process can be expressed as

$$
\frac{\Gamma\left(\mathrm{K}^{+}+\pi^{+} \nu \bar{\nu}\right)}{\Gamma\left(\mathrm{K}^{+}+\mathrm{ALL}\right)} \lesssim 5 \times 10^{-12} \mathrm{~N}_{\nu}
$$

The exciting point is that the ratio depends on the number of neutrino types $N_{V}$ present in nature. The ratio is not simply related to $N_{v}$, however, as the coefficlent in front of $N_{\nu}$ depends also on the masses of thelr associated charged leptons.

The limit on this ratio is $6 \times 10^{-7}$, which comes from an experiment done at the Bevatron by Cable et al. ${ }^{13}$ They stopped $1.1 \times 10^{9} \mathrm{k}^{+}$in a carbon target surrounded by a scintillator and lead-glass array and looked for the decay sequence

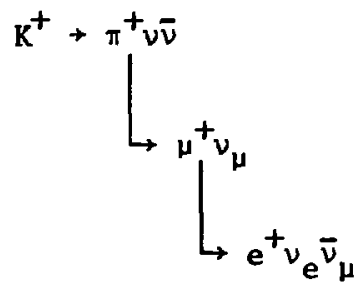

No such decays were observed.

IV. DECAYS THAT ARE SENSITIVE TO NEW INTERACTIONS

The WS gauge model of the electroweak interaction has been successful in accounting for the presently available data. In this model the left-handed leptons and quarks are grouped into SU( $\angle)$ doublets 


$$
\left(\begin{array}{l}
\nu_{e}^{\prime} \\
e
\end{array}\right)_{L} \quad\left(\begin{array}{c}
\nu_{\mu}^{\prime} \\
\mu
\end{array}\right)_{L} \quad\left(\begin{array}{c}
\nu_{\tau}{ }^{\prime} \\
\tau
\end{array}\right)_{L} \text { o0 } 0
$$

and

$$
\left(\begin{array}{l}
u \\
d^{\prime}
\end{array}\right)_{L} \quad\left(\begin{array}{l}
c \\
s^{\prime}
\end{array}\right)_{L} \quad\left(\begin{array}{l}
t \\
b^{\prime}
\end{array}\right)_{L} \quad 000
$$

whereas the right-handed particles are contained in the SU(2) singlets. These particles interact electroweakly with the spin-1 gauge particles:

$$
\mathrm{w}^{ \pm}, \mathrm{z}^{\mathrm{o}}, \mathrm{r}
$$

The $\mathrm{W}^{ \pm}$interacts with the left-handed doublets, the $\mathrm{Z}^{\circ}$ interacts with left-handed doublets and the right-handed singlet;, and the $y$ interacts with the charges. The quarks interact strongly through gauge particles called vector gluons as described by the quantum chromodynamics (QCD) theory. In addition, at least one Higgs doublet is required with its accompanying physical neutral particle to ensure renormalizability and to give mass to the fermions and bosons.

The theory puts no constraints on the number, mass, or couplings of these doublets. If more than one doublet exists, then muon-violating decays can occur.

In general, the gauge-group eigenstates $\nu_{e}{ }^{\prime}, \nu_{\nu}{ }^{\prime}, \nu_{\tau}^{\prime}$ and $d^{\prime}, s^{\prime}, b^{\prime}$, which appear in the $S U(2)$ doublets, do not correspond to the physical particles $v_{e}$, $\nu_{\mu}, v_{\tau}$ and $d, s, b$. If there are only six quarks, che gauge-group eigenstates $d^{\prime}, s^{\prime}, b^{\prime}$ are related to the physical particles $d, s, b$ via the Kobayaski-Maskawa (KM) mixing matrix: ${ }^{14}$

$$
\begin{aligned}
& \left(\begin{array}{c}
d^{\prime} \\
s^{\prime} \\
b^{\prime}
\end{array}\right)=\left(\begin{array}{ccc}
c_{1} & s_{1} c_{3} & s_{1} s_{3} \\
-s_{1} c_{2} & c_{1} c_{2} c_{3}-s_{2} s_{3} e^{i \delta} & c_{1} c_{2} s_{3}+s_{2} c_{3} e^{i \delta} \\
s_{1} s_{2} & -c_{1} s_{2} c_{3}-c_{2} c_{3} e^{i \delta} & -c_{1} s_{2} s_{3}+c_{2} c_{3} e^{i \delta}
\end{array}\right)\left(\begin{array}{c}
d \\
s \\
b
\end{array}\right) . \\
& c_{i}=\cos \theta_{i} \quad s_{i}=\sin \theta_{i} \quad t=1,2,3
\end{aligned}
$$


where $\theta_{1}, \theta_{2}$, and $\theta_{3}$ are three real mixing angles and $\delta$ is a CP-violating phase parameter.

In the WS model, the neutrinos are massless. Accordingly, lepton-flavor changing interactions cannot occur. With a finite neutrino mss, however, mixing of leptons similar to the mixing of the quarks may happen. If this is the situation, decays that violate mon-number conservation can occur. The experimental limits on the $v_{e}, v_{\mu}$, and $v_{\tau}$ masses limit the rates for such processes. For example, Herczeg ${ }^{15}$ has placed the following linits

$$
\frac{\Gamma\left(K^{+}+\pi^{+} \mu e\right)}{\Gamma\left(K^{+}+a 11\right)}=<7 \times 10^{-18}
$$

and

$$
\frac{\Gamma\left(\mathrm{K}_{\mathrm{L}}+\mu \varepsilon\right)}{\Gamma\left(\mathrm{K}_{\mathrm{L}}+\mathrm{a} 11\right)}=<5 \times 10^{-16}
$$

if only neutrino mixing happens. The search for mon-number-violating decays like these can thus be used to probe for the existence of other new Interactions down to small levels.

Now the particles in each generation of leptons and quarks

$$
\begin{aligned}
& \left(\begin{array}{l}
v_{e} \\
e
\end{array}\right)_{L} 000 \\
& \left(\begin{array}{l}
u \\
d^{\prime}
\end{array}\right)_{L}
\end{aligned}
$$

are all coupled to the same gauge bosons, all fit into the sane wS nodel, and a 11 have the same QCD Interactions among the quarks. Although the WS model agrees quantitatively with the available data, it does not answer the question of why the higher mass generations 


$$
\begin{array}{ll}
\left(\begin{array}{l}
\nu_{\mu}{ }^{\prime} \\
\mu
\end{array}\right) & \left(\begin{array}{l}
v_{\tau} \\
\tau
\end{array}\right) \\
\left(\begin{array}{l}
c \\
s^{\prime}
\end{array}\right) & \left(\begin{array}{l}
t \\
b^{\prime}
\end{array}\right)
\end{array}
$$

exist or why they have the masses they possess. Although no experimental Inconsistency with the WS model exists, the redundancy of quark and lepton flavor suggests the existence of a new horizontal symmetry that relates and distingutshes the difference generations. This symmetry may be a local gauge symmetry, in which case it leads to the existence of new gauge bosons and associated interaction. The bosons would be neutral and have flavor-changing couplings to fermions.

In the model of Cahn and Harari ${ }^{16}$, decays such as

$$
\mathrm{K}^{+}+\pi^{+} \mu^{+} \mathrm{e}^{-} \text {or } \mathrm{K}_{\mathrm{L}} \rightarrow \mu^{+} \mathrm{e}^{-}
$$

could proceed via a horizontal-generation-changing boson $H^{\circ}$ of mass $\mathrm{M}_{\mathrm{H}}$ with coupling $\mathrm{G}_{\mathrm{H}}$

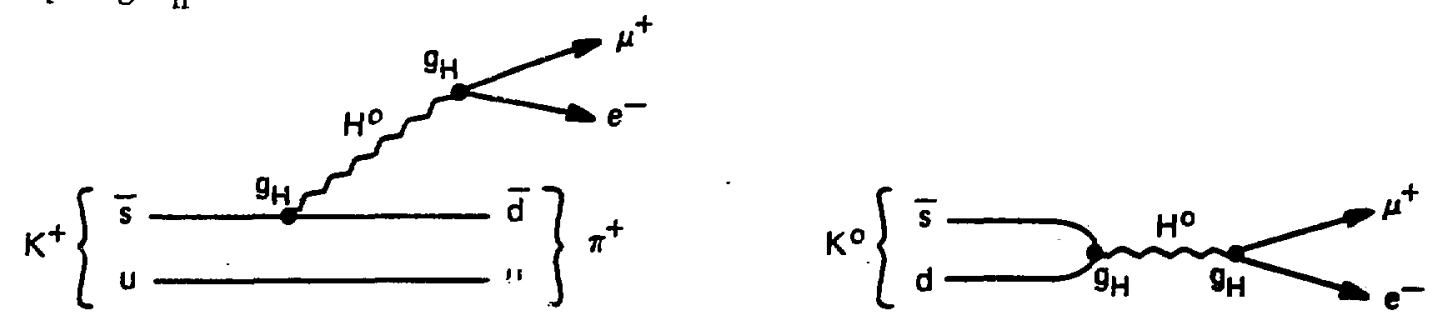

The experimental limit on the decay $\mathrm{K}^{+}+\pi^{+}{ }_{\mu}{ }^{+} \mathrm{e}^{-}$provides the following linit on $\mathrm{M}_{\mathrm{H}}$ and $\mathrm{g}_{\mathrm{H}}$ :

$$
\begin{gathered}
\frac{\Gamma\left(\mathrm{K}^{+}+\pi^{+} \mu^{+} \mathrm{e}^{-}\right)}{\Gamma\left(\mathrm{K}^{+}+\pi^{0}{ }^{+}{ }_{v_{\mu}}\right)}=\left(\frac{\mathrm{g}_{\mathrm{H}}^{2}}{2 \mathrm{~K}_{\mathrm{N}}^{2}}\right)^{2} \frac{16}{\sin ^{2} \theta_{\mathrm{c}}}\left(\frac{2 \mathrm{M}_{\mathrm{W}}^{2}}{\mathrm{~g}_{\mathrm{W}}^{2}}\right)^{2}<1.5 \times 10^{-7} \\
\mathrm{M}_{\mathrm{H}}>18 \mathrm{TeV} / \frac{\mathrm{g}_{\mathrm{H}}}{\mathrm{g}_{\mathrm{W}}}
\end{gathered}
$$


Here, $g_{w}$ is the electroweak coupling constant and $M_{w}$ is assumed equal to $85 \mathrm{GeV}$. Similarly, the limit on the decay $K_{L}+\mu^{+} e^{-}$provides the following inequalities

$$
\begin{gathered}
\frac{\Gamma\left(\mathrm{K}_{\mathrm{L}}+\mu^{+} \mathrm{e}^{-}\right)}{\Gamma\left(\mathrm{K}^{+}+\mu^{+} \nu_{\mu}\right)}=\left(\frac{\mathrm{g}_{\mathrm{H}}^{2}}{2 \mathrm{M}_{\mathrm{H}}^{2}}\right)^{2} \frac{1}{\mathrm{sin}^{2} \theta_{\mathrm{c}}}\left(\frac{2 \mathrm{M}_{\mathrm{W}}^{2}}{\mathrm{BW}^{2}}\right)^{2}<0.6 \times 10^{-9} \\
\mathrm{M}_{\mathrm{H}}>36 \mathrm{TeV} \mid \frac{\mathrm{g}_{\mathrm{H}}}{\mathrm{g}_{\mathrm{W}}} .
\end{gathered}
$$

The bounds Imposed on $\mathrm{M}_{\mathrm{H}}$ by processes such as

$$
\begin{aligned}
& \mu-\mathrm{N} \rightarrow \mathrm{e}^{-\mathrm{N}} \\
& \mu \rightarrow 3 e \\
& \mu \rightarrow e^{-} \\
& \mathrm{K}_{\mathrm{S}}-\mathrm{K}_{\mathrm{L}} \text { mass difference }
\end{aligned}
$$

are more model dependent than those given above.

A reasonable assumption is that $1^{b_{H}} /^{b_{W}} \sim 1$. Accordingly a lower limit of 10 to $100 \mathrm{TeV}$ can be placed on $\mathrm{M}_{\mathrm{H}}$.

If improved measurement of the above two processes were to show nonvanishing branching ratios, a whole new area of physics would be opened! Even if no real effects are discovered, improvements of the experimental limits would lead to better bounds on $\mathrm{M}_{H^{*}}$ Raising the linit on $\mathrm{M}_{H}$ beyond $100 \mathrm{TeV}$ would place significant constraints on future models.

As was mentioned earlier, muon number can also be violated through Higgs exchange. Branching ratios for the above two processes can be as large as the present experimental 1imits. In the model of Herczeg, ${ }^{1}$ these 1 imits force the mass of the extra Higgs bosor to be greater than $320 \mathrm{GeV}$.

Spinless lepto-quarks (bosons causing quark-lepton transitions) appear in extended technicolor models. They, in addition, could give rise to branching ratios for the above two processes near their present 1imit. 15,17 In this case, the lepto-quark exchange is not constrained significantly by the $K_{S}-K_{L}$ mass difference. 
Other decays such as

$$
\begin{aligned}
& k_{L}+\pi^{o} \mu e \\
& K_{S}+\pi^{o} \mu e \\
& K_{S}+\mu e
\end{aligned}
$$

violate muon-number conservation. In the schemes considered above, the rates for these processes are expected to be extremely los, owing to the experimental bounds on $\mathrm{K}^{+} \rightarrow \pi^{+} \mu \mathrm{e}$ and $\mathrm{K}_{\mathrm{L}} \rightarrow \mu e$.

$\underline{\mathbf{k}^{+}+\pi^{+} \mu \mathrm{e}}$

Experimentally, $\mathrm{K}^{+}+\pi^{+} \mu \mathrm{e}$ has been searched for by a group at the CERH-PS using a $2.8-\mathrm{GeV} / \mathrm{c}$ beam of $\mathrm{K}^{+}$mesons. 18 The experiment was part of a larger effort to measure $\mathrm{Ke}_{4}$ decays. Their apparatus consisted of two Cerenkov counters to identify the electron or positron and a bending magnet with MWC and spark chambers to measure the momenta of the decay products. Downstrean of the last Cerenkov counter was a lead-scintillation counter hodoscope that was used to detect $\gamma$ rays. The trigger required three charged particles with at least one being an electron or positron.

One event of the type $\mathrm{K}^{+}+\pi^{ \pm} \mu^{\mp} \mathrm{e}^{+}$was observed. For kaon decay into a positron they expected a background of $\hat{i} .3$ events and for kaon decay into an electron they expected 0.1 events due to

$$
\begin{aligned}
& \mathrm{k}^{+}+\pi^{+} \pi^{\circ} \\
& \mathrm{re}^{+} \mathrm{e}^{-} \\
& \mathrm{K}^{+}+\pi^{+} \pi^{-} \mathrm{e}^{+}
\end{aligned}
$$

decays. The 11 imit on branching ratio was made by comparison with the $\operatorname{Ke}_{4} 6$ gignal observed. Using

$$
\begin{aligned}
\operatorname{Br}\left(\mathrm{Ke}_{4}\right) & =(3.7 \pm 0.2) \times 10^{-5} \\
\mathrm{~N}_{\mathrm{Ke}_{4}} & =30101
\end{aligned}
$$


together with

$$
B R(K+\pi \mu e)=B R\left(K_{4}\right) \frac{N(K+\pi \mu e)}{N\left(K_{4}\right)} \cdot \operatorname{EFF,}
$$

where EFF is the relative detection efficiency for $\mathrm{K} \rightarrow \pi$ e with respect to $\mathrm{Ke}_{4}$, the following limits were set:

$$
\begin{aligned}
& \mathrm{BR}\left(\mathrm{K}^{+}+\pi^{ \pm} \mu^{\mp} \mathrm{e}^{+}\right)<6.9 \times 10^{-9} \\
& \mathrm{BR}\left(\mathrm{K}^{+}+\pi^{+} \mu^{+} \mathrm{e}^{-}\right)<4.8 \times 10^{-9} .
\end{aligned}
$$

$\mathrm{K}_{\mathrm{L}} \rightarrow$ He

$K_{L}+\mu e$ has been searched for at the Bevatron using a beam of $6 \times 10^{5} K_{L}$ in the momentum band $0.8<\mathrm{P}_{\mathrm{K}_{\mathrm{L}}}<3.2 \mathrm{GeV} / \mathrm{c}$ per $6 \times 10^{11}$ protons on target. 19 Electrons were identified in a 2.3-m-long Freon Cerenkov counter and the mons were identified by range measurements in a sandwich of steel and scintillator. The trigger required particles in both arms of the spectrometer. About $10^{6} \mathrm{~K} L+\pi^{+} \pi^{-}$decays were observed with a mass resolution of $1.1 \mathrm{MeV} / \mathrm{c}$. No $e^{ \pm}{ }_{\mu}^{\mp}$ events were found within $7 \mathrm{MeV}$ of the $k_{L}$ mass peak.

The branching ratio for $K_{L}+\mu$ was determined relative to the $K_{L}+\pi_{\pi}^{+}$ signal measured. The result was the $11 \mathrm{mit}$

$$
\frac{\Gamma\left(K_{L}+\mu^{ \pm} e^{\mp}\right)}{\Gamma\left(K_{L}\right.}+\frac{a 11)}{a 11.57 \times 10^{-9}}
$$

\section{SUMMARY}

As the examples of specific experiments have demonstrated, the present measurements of the rare decays of the kaon are primarily limited by adequate flux. The two- to three-order-of magnitude increase in kaon intensity. expected at LAMPF II over present day machines will enable experinenters to study realistically the processes discussed. These studies will advance the understanding of the electroweak interaction to a new level. 
The study of nonexotic decays such as

$$
\begin{aligned}
& \mathrm{K}^{+}+\pi^{+}{ }_{\nu \bar{\nu}} \\
& \mathrm{K}_{\mathrm{L}}+\mu^{+}{ }^{-}
\end{aligned}
$$

Is sensitive to effects of high-order terms in the electroweak pnteraction. Measurements of these reactions provlde cruclal tests of the wS model. On the other hand, the study of exotic decays such as

$$
\begin{aligned}
& \mu^{+}+\pi^{+} \mu^{+} \mathrm{e}^{-} \\
& \mathrm{K}_{\mathrm{L}}+\mu^{+} \mathrm{e}^{-}
\end{aligned}
$$

is sensitive to new flavor-changing neutral gauge losons, Higgs particles, or 1epto-quarks. The observation of these decays offers a unique way of probing. the physics in the multi-TeV region.

In conclusion, the study of the rare decays of the kaon ls both consolidating and exploratory in purpose. For both classes, their detailed study will advance greatly our understanding of the basic íorces of nature.

\section{ACKNOWLEDGAENTS}

I would like to thank David Bowman, Terrence Goldman, Peter Herczeg, and Cycus Hof fman for my stimulating discussions concerning the contents $r \dot{r}$ this paper. 


\section{REFERENCES}

1. H. Harari, "Quarks and Leptons," Physics Reports 42C, 235 (1978).

2. J. R. Sanford and C. L. Wang, "Empirical Formulas for Particles Production In P-Be Collisions between 10 and $35 \mathrm{BeV} / \mathrm{C}$, Part II," Brookhaven National Laboratory report BNL-11479 (May 1967).

3. G. M. Bunce, "AGS Beams - May 1978," Brookhaven National Laboratory report BNL-50874 (June 1978).

4. T.W.L. Sanford, "Number of Detectable Kaon Decays at LAMPF II," Los Alamos National Laboratory report LA-9334-MS (Apr1]. 1982).

5. S. Gasiorowicz, Elementary Particle Physics (John Wiley and Sons, Inc., New York, 1966), pp.520-558.

6. J. Heintze et al., "A New Measurement of the $\left(\mathrm{K}^{+} \rightarrow \mathrm{e}^{+} v\right) /\left(\mathrm{K}^{+} \rightarrow \mu^{+} \nu\right)$ Branching Ratio," Phys. Lett. 60B, 302 (1976).

K. S. Heard et al., "A Measurement of the $\left(\mathrm{K}^{+}+\mathrm{e}^{+} \mathrm{v}\right) /\left(\mathrm{K}^{+} \rightarrow \mu^{+} \mathrm{v}\right)$ Branching Ratio," Phys. Lett. 55B, 327 (1975).

7. T. Goldman and W. J. Wilson, "Radiative Corrections to Leptonic Decays of Charged Pseudoscalar Mesons," Phys. Rev. D 15, 709 (1977).

8. J. Heintze et al., "An Experimental Investigation of the Radiative Structure Decay $\mathrm{K}^{+} \rightarrow \mathrm{e}^{+} \mathrm{v \gamma}, "$ Nucl. Phys. B149, 365 (1979).

K. S. Heard et al., "A Measurement of $\mathrm{K}^{+}$evr Decay," Phys, Lett. 55B, 324 (1975).

9. Particle Data Group, Rev. Mod. Phys. 52 (Apri1 1980).

10. A. S. Carrol et al., "Observation of the Dalitz Decay Modes of the $R_{L}^{\circ}$," Phys. Rev. Lett. 44, 525 (1980).

11. M. K. Gaillard and B. Lee, "Rare Decay Modes of the $K$ Meson In Gauge Theories," Phys. Rev. D 10, 897 (1974).

12. E. Ma and J. Okada, "Progresses Concerning the Number of Neutrino Types," Phys. Rev. D 18, 4219 (1978).

13. G. Cable et al., "Search for Rare $\mathrm{K}^{+}$Decays. II $\mathrm{K}^{+} \rightarrow \pi^{+} v \bar{v}, "$ Phys. Rev. D 8, 3807 (1973).

C. Y. Pang et al., "Search for Rare $\mathrm{K}^{+}$Decays. I $\mathbf{k}^{+}+\mu^{+} v \bar{v} v, "$ Phys. Rev. D 8, 1989 (1973).

14. M. Kobayasht and K. Maskawa, Prog. Theor. Phys. 49, 652 (1973). 
15. P. Herczeg, "On Muon Number Violating Decay," Proceedings of the Workshop on Nuclear and Particle Physics at Energies up to $31 \mathrm{GeV}$ (January 1981) 58.

16. R. N. Cahn and H. Harari, "Bounds on the Masses of Neutral Generation-Changing Gauge Bosons," Nucl. Phys. B176, 135 (1980).

17. P. Herczeg, "Symmetry-Violating Kaon Decays," Proceedings of the Kaon Factory Workshop (August 1979) 20.

18. A. M. Diamant-Berger et a1., "Study of Some Rare Decays of the $\mathrm{K}^{+}$Meson," Phys. Lett. 62B, 485 (1976).

P. Block et al., "Observation of the $\mathrm{K}^{+} \rightarrow \pi^{+} \mathrm{e}^{+} \mathrm{e}^{-}$Decay," Phys. Lett. $\underline{56 \mathrm{~B}}$, 201 (1975).

R. Cence et a1., "Search for the Rare Decay $\mathrm{K}^{+}+\pi^{+} \mathrm{e}^{+} \mathrm{e}^{-}, "$ Phys. Rev. D 10 , 776 (1974).

19. A. R. Clark et al., "Experimental Limits on the Vecays $K_{L}^{O} \rightarrow \mu^{+} \mu^{-}, e^{+} e^{-}$, and $\mu^{ \pm} \mathrm{e}^{\mp}, "$ Phys. Rev. Lett. $\underline{26}, 1667$ (1971). 


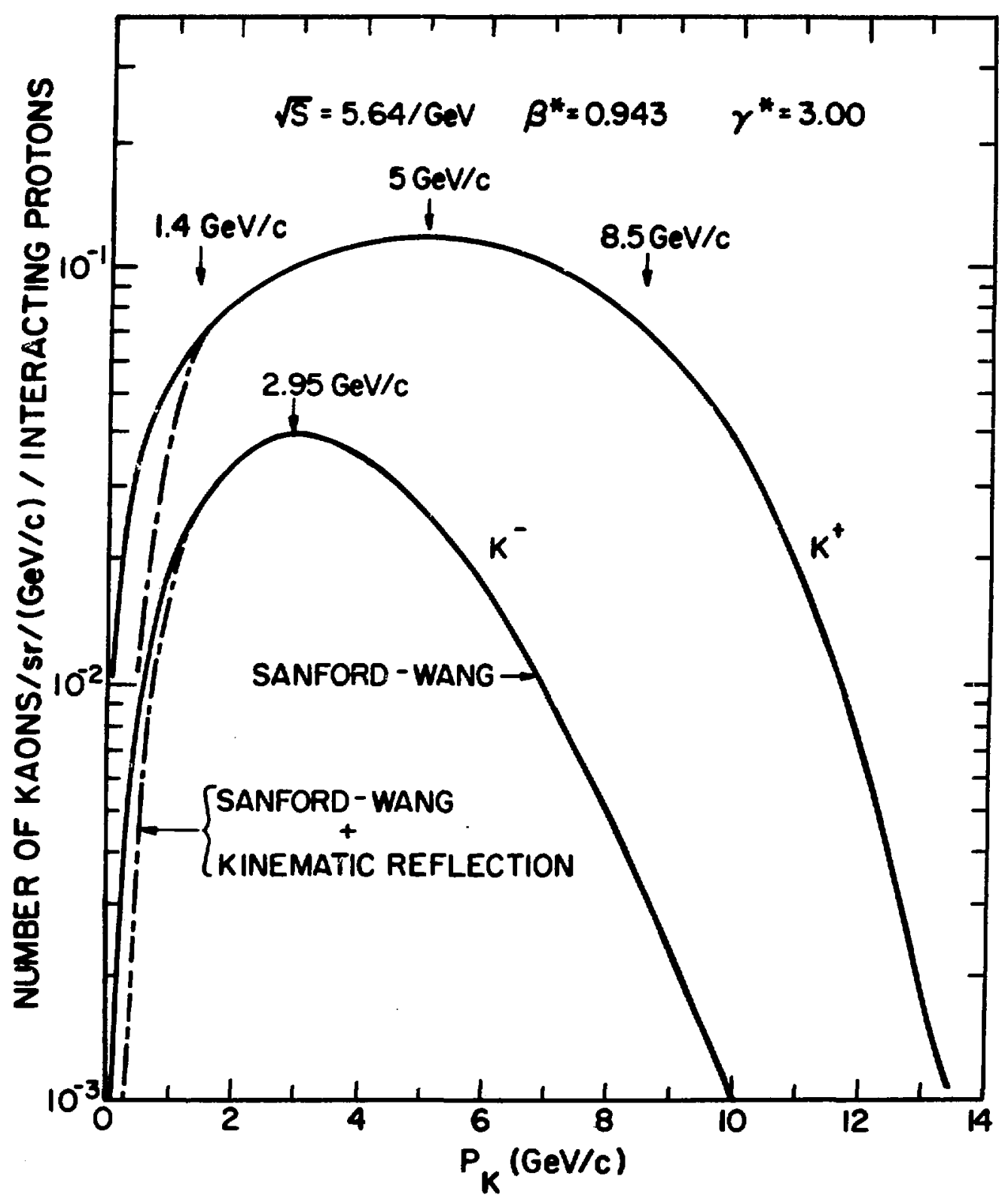

F18. 1. Kaon production cross section at $0^{\circ}$ in $\mathrm{p}-\mathrm{Be}$ collisions at $16 \mathrm{GeV} / \mathrm{c}$. 